\title{
Tele-Medicine Services in Hematological Practice During Covid Pandemic: Its Feasibility and Difficulties
}

\author{
Pradeep Kumar $^{1} \cdot$ Mukul Aggarwal $^{1} \cdot$ Rishi Dhawan $^{1} \cdot$ Jasmita Dass $^{1}$ • \\ Ganesh Kumar $^{1}$ - Vishnu Sharma ${ }^{1}$. Saleem Mirza ${ }^{1}$. Jayastu Senapati ${ }^{1}$. \\ Neha Ganju ${ }^{1}$ - Tejasvini Vaid ${ }^{1}$ Mona Vijayran ${ }^{1}$. Tribikram Panda ${ }^{1}$. \\ G Stitha Pragna ${ }^{1} \cdot$ Sahitya Sri Krishna $^{1} \cdot$ Akash Khandelwal $^{1} \cdot$ Renjith Verghese $^{1}$. \\ Seema Tyagi $^{1} \cdot$ Tulika Seth $^{1} \cdot$ Manoranjan Mahapatra $^{1}$
}

Received: 8 October 2020/Accepted: 12 November 2020/Published online: 20 November 2020

(C) Indian Society of Hematology and Blood Transfusion 2020

\begin{abstract}
In COVID 19 pandemic, delivery and access of health care services have become challenging. Telemedicine services can be considered for management of patients with hematological diseases. This study included all patients who enrolled for telemedicine facility for hematology from May 15 to July 15, 2020. Patient's demographic and disease related parameters were recorded during the teleconsultation call. Overall satisfaction of attending doctor and patients were also recorded. A total of
\end{abstract}

1187 teleconsultation appointments were taken, of which $944(79.6 \%)$ were successfully attended. Median age of patients was 38 years (range- $0.5-78$ years), with $38 \%$ females. $55 \%$ of successful calls were from patients suffering a malignant hematological disorder. $24 \%$ had an active complaint pertaining to their disease or treatment. Of these, $162(17 \%)$ were asked for a physical consultation. A significant association was found between the requirement of physical consultation and diagnosis $(p<0.001)$,

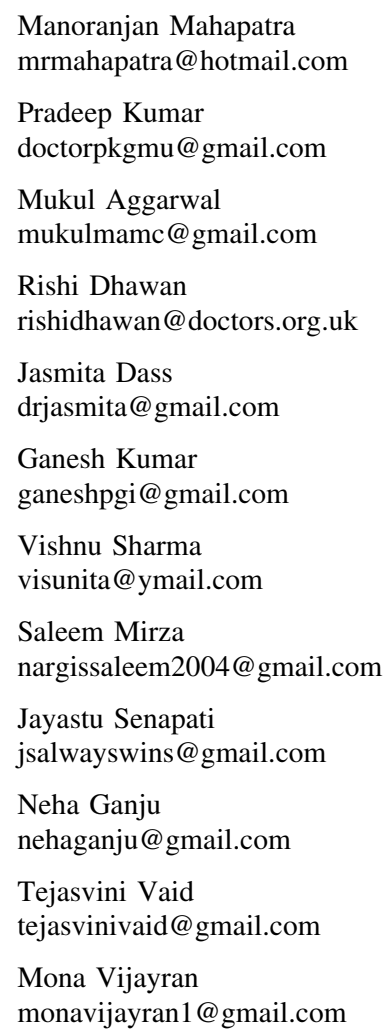

Tribikram Panda

tribikramvss@gmail.com

G Stitha Pragna

gstithap@gmail.com

Sahitya Sri Krishna

balu.sahitya@gmail.com

Akash Khandelwal

doc.akash22@gmail.com

Renjith Verghese

renjith.verghese@gmail.com

Seema Tyagi

drseematyagi@hotmail.com

Tulika Seth

drtulikaseth@gmail.com

Department of Hematology, All India Institute of Medical Sciences, New Delhi, India 
absence of active complaint $(<0.0001)$ and education level of responder $(p=0.008)$. Patients understand that teleconsultation is helpful in preventing COVID-19 infection (71.4\%) and avoids outpatient department rush (14.5\%) associated with physical appointments; and around $80 \%$ patients were satisfied with the teleconsult. With the emergence of COVID 19, many localities under partial lockdown with constant fear of contacting virus amongst patients and health care providers, we can clearly see the advantages as well as feasibility of telemedicine services for our patients. The acute surge in telemedicine could be harnessed in the future to provide comprehensive and integrated care to patients of hematological disorders.

Keywords COVID 19 pandemic - Telemedicine . Hematological diseases

\section{Introduction}

During the COVID 19 pandemic, both health care access and delivery have become particularly challenging. Physical distancing is the most vital and effective way reducing the risk of infection [1,2]. Efforts should be made not only to reduce patient visits to hospitals, but also to minimize their contact with the health care workers. Patients with hematological disease have been considered at high risk for severe COVID 19 infection [3, 4]. Many of the patients with hematological disorders are immunocompromised; need consistent and regular access to medical opinion for treatment modifications and supportive therapy. This includes prescription refills, essential medications, close follow up. The pandemic brought many challenges which has both the patient and doctor perspectives. Patients may need information on COVID related symptoms, impact of COVID on their disease, need for early institution/deferment of therapy etc. [4].

Telemedicine is a branch of science which used electronic communication or audiovisual technologies for exchange of medical information from one location to another [5], with aim to improve patient health care. These technologies (audio-visual or audio only) can be utilized for new or follow up patients with hematological diseases. During the COVID-19 pandemic, our tertiary care hospital started teleconsultation services for its patients who were not able to attend the physical outpatient department. The patients took the teleconsultation appointment online and doctors called the patients on telephone number provided on the day of appointment. We carried out this study to understand the feasibility of telemedicine services for patients with various hematological disorders, and to understand various factors responsible for a successful teleconsultation.

\section{Material and Methods}

This study included all patients who enrolled for telemedicine facility for hematology during the period from May 15 to July 15, 2020 at a government hospital based in North India. This is a tertiary care referral center with over 1500 bed capacity. Patients who were not able to attend telemedicine call for any reasons were excluded from the analysis. The patient's demographic and disease related parameters were recorded during the teleconsultation call.

Those patients who required physical visit to the hospital for any reason were requested to visit the Hematology Daycare or hospital emergency depending on the urgency. If they were not able to visit hospital, they were asked to visit a local medical facility. The doctor attending the teleconsultation were asked to provide a satisfaction score on the overall interaction with responder, on a scale of 1-5, 1 being least satisfied and 5 being fully satisfied. In addition, a feedback form on teleconsultation services was sent to all patients by a SMS link to a google forms and their responses were analyzed.

The data was tabulated and analyzed on Microsoft Excel (version 14.1.0). A $p$ value of $<0.05$ was considered significant. Institutional ethical clearance was obtained to carry out the study.

\section{Results}

During the study period, a total of 1187 teleconsultation appointments were taken, of which 944 (79.6\%) were successfully attended. One fifth of the successful calls were from patients who have been attended previously on teleconsultation.

The median age of the patients was 38 years (range$0.5-78$ years), with $38 \%$ females. Baseline diagnosis, duration of follow up, education status of patients/responders, satisfaction scores are mentioned in Table 1. Around $55 \%$ of successful calls were from patients suffering a malignant hematological disorder. Almost three-fourth of patients $(n=702,74.3 \%)$ were for regular follow up and needed advice for drug dose modifications or continuation of therapy. Around $24 \%$ had an active complaint pertaining to their disease or treatment. Of these, $162(17 \%)$ were asked for a physical consultation. Out of the successful calls, majority of patients were of chronic myeloid leukemia $(29.3 \%)$, acquired or inherited marrow failures (17\%), myeloma or lymphoma (10.1\%) and anemia's including hemolytic anemia $(9.9 \%)$ because of their stable disease and medications without major modifications. 
Table 1 Baseline parameters of tele-consultations

\begin{tabular}{|c|c|}
\hline Parameter & $N(\%)$ \\
\hline Call outcome & $944(79.6 \%)$ \\
\hline Successful & $243(20.4 \%)$ \\
\hline Unsuccessful & $93(7.8 \%)$ \\
\hline Contact number unreachable & $79(6.7 \%)$ \\
\hline Calls unanswered & $29(2.4 \%)$ \\
\hline Wrong contact number & $29(2.4 \%)$ \\
\hline Contact number unavailable & $13(1.1 \%)$ \\
\hline \multicolumn{2}{|l|}{ Miscellaneous } \\
\hline Duration of follow Up & $24(0-250)$ \\
\hline Median (Range) in months & $100(10.6 \%)$ \\
\hline \multicolumn{2}{|l|}{ Data not Available } \\
\hline Education Level & $23(2.4 \%)$ \\
\hline No Formal education & $43(4.5 \%)$ \\
\hline Till Primary education & $259(27.4 \%)$ \\
\hline Primary-secondary education & $267(28.3 \%)$ \\
\hline Graduation and above & $352(37.3 \%)$ \\
\hline \multicolumn{2}{|l|}{ Data not available } \\
\hline Diagnosis & $69(7.3 \%)$ \\
\hline ALL/MPAL/APML & $39(4.1 \%)$ \\
\hline AML/MDS & $277(29.3 \%)$ \\
\hline CML & $56(5.9 \%)$ \\
\hline MPN/CMPD/ET/PV/MF/HES & $94(9.9 \%)$ \\
\hline Anemia including hemolytic anemia & $161(17 \%)$ \\
\hline AA/PRCA/Fanconi & $57(6 \%)$ \\
\hline ITP/TTP & $21(2.2 \%)$ \\
\hline Undiagnosed & $96(10.1 \%)$ \\
\hline Myeloma/Lymphoma & $56(5.9 \%)$ \\
\hline DVT/ Coagulation disorders & $18(1.9 \%)$ \\
\hline \multicolumn{2}{|l|}{ Not Available } \\
\hline Satisfaction of doctor score & $32(3.4 \%)$ \\
\hline Score 1 (Least satisfied) & $46(4.8 \%)$ \\
\hline Score 2 & $208(22 \%)$ \\
\hline Score 3 & $344(36.4 \%)$ \\
\hline Score 4 & $298(31.5 \%)$ \\
\hline Score 5 (Highly satisfied) & $16(1.7 \%)$ \\
\hline Not available & \\
\hline
\end{tabular}

\section{Factors for Successful Teleconsultation}

A significant association was found between the requirement of physical consultation and diagnosis $(p<0.001)$, absence of active complaint $(<0.0001)$ and education level of responder $(p=0.008)$ (Table 2). Mean duration of follow up of patients needing physical consultation was $25.1 \pm 33.2$ months vs. $46.04 \pm 46.4$ months for those who did not need physical consultation $(p=<0.001)$. Age, sex and nature of disease (benign/malignant) were not significantly associated with it. Patients of acute leukemia, myeloma, lymphoma and undiagnosed patients, those who had an active complaint, lower education status and smaller duration of follow up were more likely to be called for physical consultation.

Satisfaction of attending doctor was significantly associated with duration of follow up ( $p=<0.0001)$, whether the call was for a regular follow up ( $p=0.025)$, need for a physical consultation $(p=0.0001)$ and education level of responder $(p=0.0001)$. So, the attending doctor were more likely to be satisfied with the quality of teleconsultation if patients did not have any active complaint, did not need a physical visit to healthcare facility and have better education status. (Table 3).

\section{Patient Feedback}

The SMS link to google form to assess patient feedback was sent to all successful teleconsultation, however, only 53 of them replied. Although majority of the patients preferred teleconsultation mode, $21.6 \%$ were not satisfied with teleconsultation. People understand that this is especially helpful in preventing COVID-19 infection (71.4\%) and avoids outpatient department rush (14.5\%) associated with physical appointments. On suggestion to improve telemedicine services, around $37 \%$ of patients wanted to talk to the doctor of their choice, $17.6 \%$ wanted video option, $17.6 \%$ wanted more time and $15.7 \%$ wanted medicine prescription being sent to them, in addition to the verbal advice (Table 4).

\section{Discussion}

COVID-19 pandemic has posed unique challenges to the healthcare delivery systems due to problems caused by lockdowns as well as need to maintain social distancing to decrease exposure [6]. With the imposition of lockdowns and restricted patient movement across cities as well as disruption of routine outpatient department care, hematology patients were at a receiving end and would have a high risk of disease recurrence/relapse if regular medical advice is not provided. Our study showed encouraging data of feasibility of telemedicine services. More than $75 \%$ of teleappointments were successfully attended. We started from scratch and physical visits were restricted to emergency or daycare only. A study from New York hospital reported that around half of their patients who could not be attended on site were contacted by telemedicine visits in early 2020 [7]. In a global survey about oncology practice during COVID-19 pandemic, over $80 \%$ participants were using telemedicine in some form or another [8]. Both the above studies highlight the virtual non-existence of telemedicine 
Table 2 Correlation of parameters with need for physical consultation

\begin{tabular}{|c|c|c|}
\hline Factor & Ratio of requirement of physical consultation (yes/total) & $p$ value \\
\hline Sex & $97 / 574(16.9 \%)$ & 0.593 \\
\hline Male & $65 / 352(18.5 \%)$ & \\
\hline \multicolumn{3}{|l|}{ Female } \\
\hline Type of disease & $65 / 383(16.9 \%)$ & 0.928 \\
\hline Benign & $87 / 521(16.7 \%)$ & \\
\hline \multicolumn{3}{|l|}{ Malignant } \\
\hline Diagnosis & $23 / 69(33.3 \%)$ & 0.001 \\
\hline ALL/MPAL/APML & $13 / 39(33.3 \%)$ & \\
\hline AML/MDS & $14 / 277(5.0 \%)$ & \\
\hline CML & $5 / 56(8.9 \%)$ & \\
\hline MPN/CMPD/ET/PV/MF/HES & $13 / 94(13.8 \%)$ & \\
\hline Anemia including hemolytic anemia & $34 / 161(21.1 \%)$ & \\
\hline AA/PRCA/Fanconi & $4 / 57(7 \%)$ & \\
\hline ITP/TTP & $8 / 21(38 \%)$ & \\
\hline Undiagnosed & $31 / 96(32.3 \%)$ & \\
\hline Myeloma/Lymphoma & $10 / 56(17.8 \%)$ & \\
\hline \multicolumn{3}{|l|}{ DVT/Coagulation disorders } \\
\hline Type of follow up & $96 / 244(39.3 \%)$ & $<0.0001$ \\
\hline Active complaint & $64 / 696(9.2 \%)$ & \\
\hline \multicolumn{3}{|l|}{ Regular } \\
\hline Education level & $9 / 22(40.9 \%)$ & 0.008 \\
\hline No formal education & $6 / 41(14.6 \%)$ & \\
\hline Till Primary & $52 / 256(20.3 \%)$ & \\
\hline Till secondary & $36 / 265(13.5 \%)$ & \\
\hline Graduation \& above & & \\
\hline
\end{tabular}

services prior to pandemic and rapid expansion over last few months.

Around one third $(37 \%)$ patients required re-appointment in the form of either physical or repeat call. The reasons of re-consultation were to review of investigations, dose alteration or side effects of medications and related to disease course like progression of disease or other complaints of patients. Some of the patients required hospital visit after successful teleconsultation for treating physician signature for their medications and reimbursement related like government beneficiaries etc. All such physical visits can be triaged using telemedicine and patients can be directed to emergency ward, COVID screening area, daycare facility or pharmacy etc. depending on the inputs from teleconsult [6].

Those patients, who need to travel a long and tiring journey, can easily approach their physician for follow up issues related to disease $\&$ treatment without any exposure risk. E-prescription through message, email etc. can be sent to them thus avoiding physical visit. This will save on man hours, decrease office/school holidays as well as avoid over-crowding at busy government hospital outpatient services. Telemedicine-based care is easier in certain group of patients such as patients with long term oral medications e.g. chronic myeloid leukemia, chronic lymphocytic leukemia, chronic immune thrombocytopenia etc. and patient in complete remission and on maintenance therapy for their disease e.g. acute lymphoblastic leukemia, multiple myeloma, aplastic anemia etc. These patients are less likely to have active complaint during their treatment; drug modifications are easier and hence less likely to need physical outpatient department visit, as reflected in our data. More than one third of acute leukemia or myelodysplasia, myeloma, lymphoma and undiagnosed patients who consulted on phone were requested for physical consultation, as reflected in our data.

Challenges in drug availability were expected, as many patients living in second/third tier cities or villages were not able to procure medicines. Similar problems have been reported in an Italian CML study, where Imatinib was easily accessible but other medicines delivery issues were faced by $36 \%$ physicians for their patients [9]. Our patients 
Table 3 Correlation of parameters with overall satisfaction of doctor from teleconsultation

\begin{tabular}{|c|c|c|c|c|}
\hline Factor & Category 1 (Score $1+2)$ & Category 2 (Score 3 ) & Category $3($ Score $4+5)$ & $p$ value \\
\hline Sex & $42 / 575$ & $123 / 575$ & $410 / 575$ & 0.143 \\
\hline Male & $36 / 353$ & $85 / 353$ & $232 / 353$ & \\
\hline \multicolumn{5}{|l|}{ Female } \\
\hline Type of disease & $29 / 379$ & $92 / 379$ & $258 / 379$ & 0.720 \\
\hline Benign & $39 / 522$ & $115 / 522$ & $368 / 522$ & \\
\hline \multicolumn{5}{|l|}{ Malignant } \\
\hline Type of follow up & $27 / 244$ & $49 / 244$ & $148 / 244$ & 0.025 \\
\hline Active & $44 / 696$ & $158 / 696$ & $494 / 696$ & \\
\hline \multicolumn{5}{|l|}{ Regular } \\
\hline Requirement of physical consultation & $29 / 160$ & $44 / 160$ & $87 / 160$ & $<0.0001$ \\
\hline Yes & $44 / 760$ & $162 / 760$ & $554 / 760$ & \\
\hline \multicolumn{5}{|l|}{ No } \\
\hline Requirement of repeat call & $23 / 187$ & $20 / 187$ & $144 / 187$ & $<0.0001$ \\
\hline Yes & $52 / 736$ & $187 / 736$ & $497 / 736$ & \\
\hline \multicolumn{5}{|l|}{ No } \\
\hline Education Level & $7 / 22$ & $5 / 22$ & $10 / 22$ & $<0.0001$ \\
\hline No formal education & $12 / 43$ & $16 / 43$ & $15 / 43$ & \\
\hline Till primary & $37 / 259$ & $77 / 259$ & $145 / 259$ & \\
\hline Till secondary & $7 / 265$ & $51 / 265$ & $207 / 265$ & \\
\hline Graduation \& above & & & & \\
\hline
\end{tabular}

Table 4 Patient feedback $(n=53)$

\begin{tabular}{ll}
\hline Preferred mode of consultation during pandemic & \\
Telemedicine appointment & $42.9 \%$ \\
Physical appointment & $12.2 \%$ \\
Both are good & $44.9 \%$ \\
Satisfaction with telemedicine services & \\
Yes & $62.7 \%$ \\
No & $21.6 \%$ \\
Somewhat satisfied & $15.7 \%$ \\
What is the major reason for telemedicine preference? & \\
Reduced travel time & $6.1 \%$ \\
Avoiding OPD rush & $14.5 \%$ \\
Especially helpful in preventing corona virus & $71.4 \%$ \\
Saves a day at work & \\
Belong to hot spot area & $2.0 \%$ \\
All of above & $2.0 \%$ \\
Other & $4.0 \%$ \\
How can telemedicine services be made better? & \\
Video option & $17.6 \%$ \\
Medicine list can be sent & $15.7 \%$ \\
Longer time devoted to us & $17.6 \%$ \\
Talking to doctor of our choice & $37.3 \%$ \\
This does not help in our patient & $7.9 \%$ \\
Other & $3.9 \%$ \\
\hline
\end{tabular}

who underwent stem cell transplant $<6$ months ago, were constantly in touch with us and always required to visit daycare facility for assessment. Only long-term follow-up transplant recipients were considered for teleconsultation. An Italian study showed that 58\% of their long term follow up patients were seen by teleconsultation. Forty nine percent long term patients were contacted by teleconsultation for disease specific information and services available [10].

Patient feedback by google form provided good insights into overall success of teleconsultation services. Overall satisfaction with teleconsultation was present and it was heartening to see that patient understand the importance of teleconsultation in COVID era and its role in preventing virus spread. Their suggestions on improving the services are easy to implement and we are in process to doing so.

One of the limiting factors for telemedicine is lack of physical examination. We suggest any patient whose symptoms or reports warrant physical examination should be called to visit nearby health care facility. In present pandemic and through telemedicine, it is difficult to break bad news in comparison to pre-COVID time. We need to develop skill and art for breaking bad news when the patient initial diagnosis is made as well as at relapse or other major complication [11]. Through telemedicine facilities patient, caregiver and relatives, staying at different places, can be counseled on a single platform as well as 
treatment plan formulated by treating physician and distantly staying specialist [6]. Video based consultation should be the preferred modality in urban and educated areas, especially with resurgence of online video platforms like zoom, Google meets, teams etc. But availability of basic infrastructure including smart phone, internet and proper surroundings for communication and education level of patients are major barrier for integration into practice. Patients of acute leukemia during induction/consolidation cycles, high grade lymphoma patients on chemotherapy, transplant recipients in early post-transplant period etc. need more supervision. Even if consulted by telemedicine, they will require physical consultations more often so that complications are managed effectively.

To conclude, with the emergence of COVID 19, many cities under partial lockdown with restricted movement of people and fear of contacting virus, we can clearly see the advantages as well as feasibility of telemedicine services for our patients. With the possibility of long persistence of COVID-19, we feel that telemedicine is here to stay and both health care providers as well as patients need to be well versed with these technologies. Various advantages associated with telemedicine and potential applications make it a feasible approach even during the non COVID times. This expansion achieved on an urgent basis could be harnessed in the future to provide comprehensive and integrated care to patients suffering from various hematological disorders.

Acknowledgements The author acknowledges Miss Jyotsna Kapoor for her valuable inputs in manuscript.

\section{References}

1. Reusken CB, Buiting A, Bleeker-Rovers C et al (2020) Rapid assessment of regional SARS-CoV-2 community transmission through a convenience sample of healthcare workers, the Netherlands, March 2020. Euro Surveill. https://doi.org/10.2807/ 1560-7917.ES.2020.25.12.2000334

2. Mian H, Grant SJ, Engelhardt M et al (2020) Caring for older adults with multiple myeloma during the COVID-19 pandemic: perspective from the international forum for optimizing care of older adults with myeloma. J GeriatrOncol. https://doi.org/10. 1016/j.jgo.2020.04.008

3. Liang W, Guan W, Chen R et al (2020) Cancer patients in SARSCoV-2 infection: a nationwide analysis in China. Lancet Oncol. https://doi.org/10.1016/S1470-2045(20)30096-6

4. Sahu KK, Jindal V, Siddiqui AD (2020) Managing COVID-19 in patients with cancer: double blow for oncologists. JCO Oncology Practice 16:223-225. https://doi.org/10.1200/OP.20.00167

5. Abdel-wahab M, Rosenblatt E, Prajogi B et al (2020) Opportunity in telemedicine, lessons learned after COVID-19 and the way into the future. Int J RadiatOncolBiolPhys. https://doi.org/10.1016/j. ijrobp.2020.07.006

6. Prateek P, Vishnoi JR, Kombathula SH et al (2020) Teleoncology: the youngest pillar of oncology. JCO Glob Oncol 6:1455-1460. https://doi.org/10.1200/GO.20.00295

7. Shah MA, Emlen MF, Shore T et al (2020) Hematology and oncology clinical care during the coronavirus disease 2019 pandemic. CA Cancer J Clin. https://doi.org/10.3322/caac.21627 (published online ahead of print, 2020 Jul 14)

8. Ürün Y, Hussain SA, Bakouny Z et al (2020) Survey of the Impact of COVID-19 on oncologist' decision making in cancer. JCO Glob Oncol. https://doi.org/10.1200/GO.20.00300

9. Breccia M, Abruzzese E, Bocchia M et al (2020) Chronic myeloid leukemia management at the time of the COVID-19 pandemic in Italy. Campus CML SurvLeuk. https://doi.org/10.1038/ s41375-020-0904-z

10. Lupo-Stanghellini MT, Messina C, Marktel S et al (2020) Following-up allogeneic transplantation recipients during the COVID-19 pandemic. Lancet Haematol. https://doi.org/10.1016/ S2352-3026(20)30176-9

11. Wolf I, Waissengrin B, Pelles S (2020) Breaking bad news via telemedicine: A new challenge at times of an epidemic. Oncologist 25:e879-e880

Publisher's Note Springer Nature remains neutral with regard to jurisdictional claims in published maps and institutional affiliations. 